\title{
Ưber Heilung von Mäuse-Ascites-Krebs durch D- und L-Glycerinaldehyd
}

\author{
Von \\ O. Warburg, K. Gawehn, A. W. Geissler und S. Lorenz \\ Aus dem Max-Planck-Institut fiir Zellphysiologie, Berlin-Dablem (Dircktor: Prof. Dr. O. Warburg)
}

(Der Schriftleitung zugegangen am 1. Oktober 1963)

\begin{abstract}
Mit ungiftigen Dosen vọn Glyccrinaldchyd kann man cinen Eurlici schen Mäuse-Ascitcs-Krebs hcilen, der niemals spontan zurückgeht. Die geheilten Tiere sind vollkommen gesund. Wir haben im vorigen Sommer 75 Tiere geheilt und dabei keine Tiere gefunden, die auf die Behandlung mit Glycerinaldchyd nicht reagieren. - Dic Heilung gelingt nicht vom Kreislauf aus, sondern nur durch Einspritzen des Aldehyds in die Bauchhöhle.
\end{abstract}

\begin{abstract}
EInRLICr mouse ascites tumour is cured by sub-toxic doses of glyceraldehyde, with no spontancous recurrences. The curcd animals are completely healthy. We cured 75 animals in the previous Summer and found none which did not react to treatment. Dosage was by intraperitoneal injection.
\end{abstract}

Im Jahre 1929 fand Bruno Mendel (1), der im KaiserWilhelm-Institut für Zellphysiologie die Messung der Tumorgärung gelernt hatte, $\mathrm{daß}$ der "unnatürliche“ L-Glycerinaldehyd die Gärung von Schnitten des JENSEN-Sarkoms der Ratte in vitro hemmt, während der "natürliche" D-Glycerinaldehyd die Gärung nicht hemmte. Von den zahlreichen darauffolgenden Arbeiten sei vor allem eine Arbeit von MEYerhof, LOHMANN und SCHUSTER (2) erwähnt, in der gezeigt wurde, da $\beta$ das Ferment Aldolase den D-Glycerinaldehyd mit Dioxyacetonphosphat zu D-Fructose-1-phosphat kondensiert, während der L-Aldehyd mit Dioxyacetonphosphat zu L-Sorbose-1-phosphat kondensiert wird. LaRDY, Wiebelhaus und MaNN (3) fügten im Jahr 1960 die Beobachtung hinzu, daß L-Sorbose-1-phosphat das Ferment Hexokinase hemmt, während D-Fructose1-phosphat die Hexokinase nicht hemmt und sahen darin die Erklärung für die Hemmung der Gärung durch Glycerinaldehyd. Der naheliegende Versuch, mit Glycerinaldehyd Krebs zu heilen, ist bereits im Jahr 1930 von Walter Schóller, dem damaligen Leiter der Forschung der Schering A. G. gemacht worden, doch scheinen die Versuche damals nicht erfolgreich gewesen zu sein. Wahrscheinlich ist Heilung durch Glycerinaldehyd bisher nur auf dem Weg über den Kreislauf versucht worden und nicht durch direkte Einwirkung des Aldehyds auf Krebszellen in vivo. Im Kreislauf aber verschwindet injizierter Glycerinaldehyd zu schnell, als daß er Tumoren abtöten könnte.

In dieser Arbeit beschreiben wir die Wirkung von $D$ und L-Glycerinaldehyd, auf das heutige Hauptversuchsobjekt der Krebsforschung, auf den EhrLICHschen Mäuse-Ascites-Krebs. Wir fanden, daß in vitro, in Salzlösungen, $0,01 \mathrm{~m}$ L-Glycerinaldehyd die Gärung von Ascites-Krebszellen sehr stark hemmt, während der D-Aldehyd unter den gleichen Bedingungen die Gärung nicht hemmte; das heißt, wir bestätigten für die Asciteskrebszellen der Maus, was MENDEL für die Schnitte des JeNsEn-Sarkoms der Ratte gefunden hatte (Abb.
1, 2 und 3). Wir fanden aber darüber hinaus, daß Krebszellen, die auf diese Weise in Manometriegefäßen in vitro $30 \mathrm{Min}$. bei $38^{\circ}$ vorbehandelt worden waren und aus denen dann der Aldehyd wieder fortgewaschen worden war, beim Impfen in die Bauchhöhle von Mäusen nicbt mehr angingen, daß also alle Krebszellen mit Glycerinaldehyd, und zwar - merkwürdigerweise - sowohl durch den L- als auch durch den D-Aldehyd abgetötet worden waren.

Und noch wichtiger: - Wir fanden, daß Glycerinaldehyd auch im lebenden Tier die Krebszellen abtötet, wenn man $50 \mu l$ Krebszellen in die Bauchhöhle impft und am selben Tag oder einen oder zwei Tage später Glycerinaldehyd in die Bauchhöhle nachspritzt. Es war dabei um so mehr Aldehyd zur Heilung notwendig, je später der Glycerinaldehyd in die Bauchhöhle nachgespritzt wurde. Nicht mit Aldehyd behandelte Tiere wurden nach 7 bis 8 Tagen infolge von Ascites-Krebs so dick, daß sie getötet werden mußten, während die mit den unten angegebenen Mengen Aldehyd behandelten Tiere nach 60 Tagen noch völlig gesund waren, und auch später nicht mehr an Krebs erkrankten. Wir haben in diesem Sommer auf diese Weise 75 Tiere geheilt. Keine Tiere wurden gefunden, bei denen die Behandlung mit Aldehyd erfolglos war. Auch bei der Abtötung in vivo zeigte sich, wie bei der Abtötung in vitro, daß $D$ - und L-Aldehyd in gleichem Maß abtöteten oder, daß der D-Aldehyd sogar wirksamer abtötete, als der L-Aldehyd. - Eine Immunität gegen die Krebszellen trat bei der Heilung nicht auf, Nachimpfungen der geheilten Tiere gingen immer in 7 bis 8 Tagen an. Vom Kreislauf aus kann man den Asciteskrebs mit Glycerinaldehyd nicbt heilen. Weder Fütterung von Glycerinaldehyd noch subkutane Applikation von Glycerinaldehyd beeinflußten die Entwicklung des Asciteskrebses. In einigen Fällen beobachteten wir, daß an der Einstichstelle in der Bauchhaut, durch die der Glycerinaldehyd in den Asciteskrebs injiziett wurde, 
Tumoren entstanden, während gleichzeitig innerkalb der Bauchhöhle die Krebszellen durch den injizierten Aldehyd abgetötet wurden. Wir werden untersuchen, aus welchem Grunde in den Kreislauf injizierter Glycerinaldehyd verschwindet. Jedenfalls geht aus der Heilung des Asciteskrebses in der Bauchhöhle hervor, daß Glycerinaldehyd für normale Zellen nicht giftig ist. Wir untersuchen zur Zeit den Einfluß des Glycerinaldehyds auf das Wachstum der embryonalen Zellen in vitro, wenn ihr Stoffwechsel durch genügend Sauerstoff normal gehalten wird oder wenn ihr Stoffwechsel wegen Mangel an Sauerstoff in Krebsstoffwechsel umgeschlagen ist.

Bei allen unsern Heilungs-Versuchen betrug die Menge an Krebszellen, die bei der Impfung in die Bauchhöhle der Mäuse eingeimpft wurde, $50 \mathrm{~mm}^{3}$ Zellen. Die Impfmenge war also sehr groß. Impfversuche mit sehr kleinen Zellmengen ergaben, daß man mit ${ }^{1} /{ }_{1000} \mathrm{~mm}^{3}$ Zellen noch mit Sicherheit Asciteskrebs erzeugen kann, allerdings mit einer Verzögerung von 10 Tagen gegenüber der Impfmenge von $50 \mathrm{~mm}^{3}$ Zellen. Heilung des Asciteskrebses bedeutete also bei unserer Versuchsanordnung, daß die Impfmenge von $50 \mathrm{~mm}^{3} \mathrm{Krebszellen}$ mindestens bis auf $1 / 1000 \mathrm{~mm}^{3}$ Krebszellen abgetötet worden sein mußte. Eine derartige Abtötung von Krebszellen im lebenden Tier, noch dazu in kurzer Zeit, durch eine ganz ungiftige Dosis eines normalen Stoffwechselprodukts ist unseres Wissens bisher noch niemals beobachtet worden und ist hier besonders bemerkenswert, weil der nicht behandelte Asciteskrebs unseres Stammes niemals spontan zurückgeht, vielmehr immer, "wwenn $50 \mathrm{~mm}^{3}$ Krebszellen !geimpft "werden, Jin kurzer Zeit zum,Tode führt.

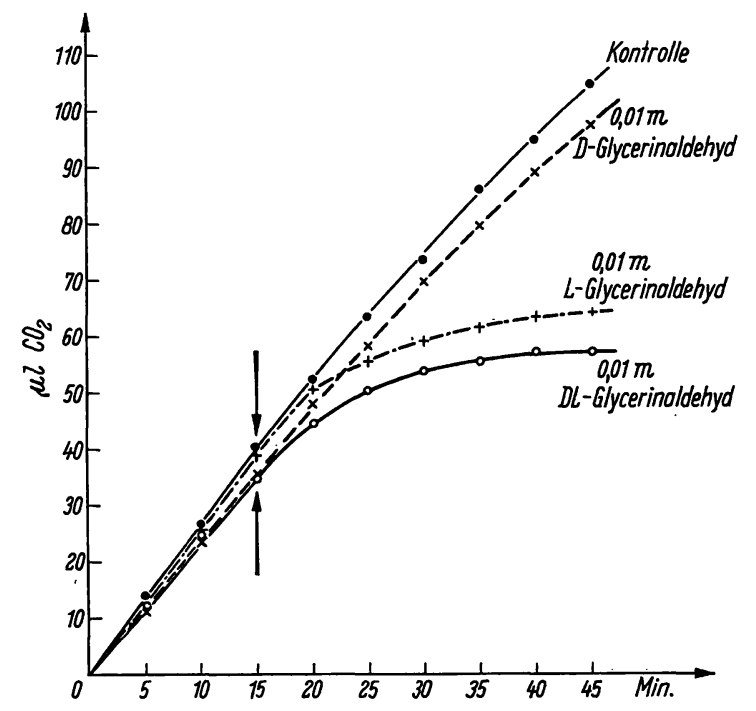

Abb. 1

Hemmung der anaeroben Gärung durch Glycerinaldehyd.

Manometriegefä $\beta$ ungefähr $20 \mathrm{~cm}^{3} \mathrm{v}_{F}=3,5 \mathrm{~cm}^{3}$, Medium wie oben beschrieben; in der Birne $30 \mu \mathrm{Mol}$ Glycerinaldehyd, der nach $15 \mathrm{Min}$. (Pfeile) in den Hauptraum eingekippt wurde. Temperatur des Thermostaten $38^{\circ}$. Gasraum: 5 Vol. $\% \mathrm{CO}_{2}$-Argon. Die Retention der Kohlensäure durch das 0,01 $m$ Phosphat wurde bestimmt und vergrößerte $\mathrm{K}_{\mathrm{co}_{2}}$ um etwa $10 \%$ ihres Werts. Zellmenge pro Gefäß $3 \mathrm{mg}$ Trockensubstanz.

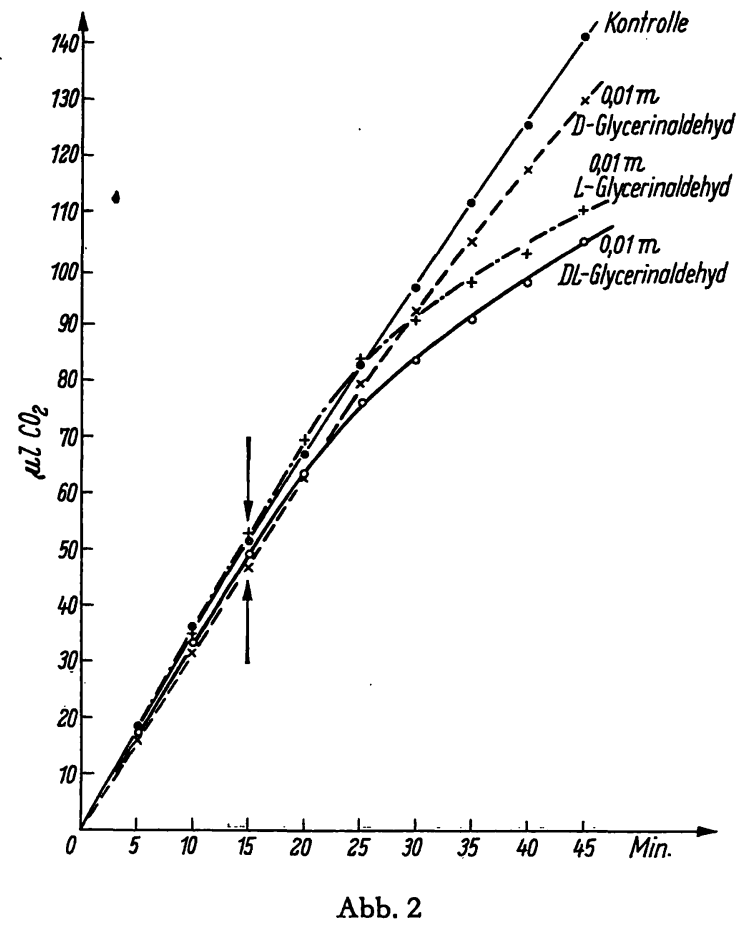

Hemmung der aeroben Gärung durch Glycerinaldehyd.

Wie in Abb. 1, aber Gastaum $5 \mathrm{Vol} . \% \mathrm{CO}_{2}$-Luft statt $5 \mathrm{Vol} . \%$ $\mathrm{CO}_{2}$-Argon und pro Gefäß $6 \mathrm{mg}$ Zellen Trockensubstanz statt $3 \mathrm{mg}$ Zellen. Die durch die Atmung erżeugten Drucke wurden mit Hilfe des Versuchs der Abb. 3 korrigiert.

Worauf die Heilung durch Glycerinaldehyd beruht, wissen wir noch nicht. Die Theorie von LARDY, die die Gärungshemmung erklären würde, kann auf die Heilung nicht angewendet werden, da der D-Aldehyd mindestens ebensogut heilt wie der L-Aldehyd.

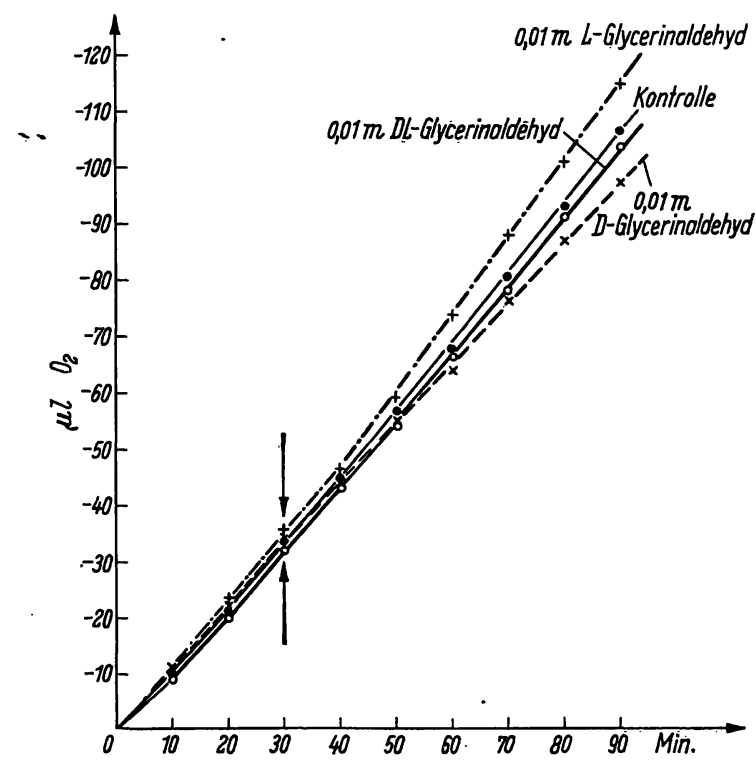

Abb. 3

Nichthemmung der Atmung durch Glycerinaldehyd.

Manometriegefäß mit Anbänger (4). Volumen etwa $26 \mathrm{~cm}^{3}, v_{F}$ im Hauptraum 3,5 cm $\mathrm{cm}^{3}$ Medium wie oben beschrieben, in der Birne $30 \mu \mathrm{Mol}$ Glycerinaldehyd, im Anhänger $2 \mathrm{mg}$ Cartase $+2,5 \mathrm{~cm}^{3}$ $3 m \mathrm{KHCO}_{3}+\mathrm{K}_{2} \mathrm{CO}_{3} ;$ Gemisch $79+21$ Volumina, wodurch ein $\mathrm{CO}_{2}$-Druck von 5 Vol. \% $\mathrm{CO}_{2}$ in der Luft des Gasraums entsteht (5). Die Zellmenge pro Gefäß betrug $12 \mathrm{mg}$ Trockensubstanz. 


\section{Versuche}

\section{Stoffinecbselversucke}

Die folgenden 3 Abbildungen geben einen Uberblick über die Wirkungen des Glycerinaldehyds auf die Atmung, die aerobe Gärung und die anaerobe Gärung von Ascites-Krebszellen der Maus. Die Krebszellen wurden zunächst in 0,9 proz. $\mathrm{NaCl}$ in $0,01 \mathrm{~m}$ Phosphat, $\mathrm{pH}=7,4$ gewaschen, dann wurden zu diesem Medium $18 \mu \mathrm{M} \mathrm{NaHCO}_{3}$ und $2 \mathrm{mg}$ Glucose pro $\mathrm{cm}^{3}$ hinzugefügt und sofort mit $5 \mathrm{Vol} . \% \mathrm{CO}_{2}$ in Luft oder inArgon gesättigt und bei $38^{\circ}$ im Thermostaten geschüttelt. Die Atmung wurde mit der neuen Eingefäßmethode (4) gemessen, bei der konzentrierte BikarbonatKarbonat-Gemische den Kohlensäuredruck von $5 \mathrm{Vol} . \%$ erzeugten und konstant hielten, so daß mit der einfachen Gleichung $x_{\mathrm{O}_{2}}=b \times k_{\mathrm{O}_{2}}$ gerechnet werden konnte. Das Ergebnis bedarf nach dem vorher Gesagten kaum eines Kommentars. Die Atmung wird durch den Glycerinaldehyd fast nicht gehemmt, die anaerobe Gärung wird durch den L-Aldehyd nach kurzer Zeit fast vollständig, durch den D-Aldehyd fast gar nicht gehemmt. Während die Hemmung der anaeroben Gärung bereits nach $15 \mathrm{Min}$. voll ausgebildet ist, werden im Fall der aeroben Gärung etwa 45 Min. benötigt.

\section{Heilungsversucke}

Das mittlere Gewicht der Mäuse betrug $25 \mathrm{~g}$, die Zellmenge, die in die Bauchhöhle geimpft wurde, betrug immer $50 \mathrm{~mm}^{3}$ Zellen, suspendiert in Kochsalz-Phosphatlösung $(0,9 \% \mathrm{NaCl}$ in $0,01 \mathrm{~m}$ Phosphat $\mathrm{pH}=7,4)$. Alle nicht behandelten Tiere wurden nach 7 bis 8 Tagen so dick, daß sie getötet werden mußten.

Die Glycerinaldehydlösung, die zwecks Heilung in die Bauchhöhle injiziert wurde, war immer 0,5 molar (Wasser oder Kochsalz-Phosphatlösung) und enthielt also $500 \mu \mathrm{Mol}$ Aldehyd pro $\mathrm{cm}^{3}$. Wurde der Aldehyd am Tage der Impfung in die Bauchhöhle injiziert, so wurden $150 \mu \mathrm{Mol}$ zur Heilung benötigt. Wurde der Aldehyd 2 Tage nach der Impfung injiziert, so wurden $300 \mu \mathrm{Mol}$ Aldehyd zur Heilung benötigt. 3 Tage nach der Impfung wurden $400 \mu \mathrm{Mol}$ Aldehyd benötigt, doch wurden 3 Tage nach der Impfung nicht immer Heilungen erzielt, so da $\beta$ man sich also auf 2 Tage nàch der Impfung beschränken sollte. $25 \mathrm{~g}$ schwerc Mäuse vertragen maximal pro Tag $1000 \mu \mathrm{Mol}$ Aldehyd, wenn sie im Lauf von $8 \mathrm{Stdn}$. eingespritzt werden. Da D-Glycerinaldehyd vielfach besser wirkte, als L-Glycerinaldehyd, so ist der D-Aldehyd bei Heilungsversuchen vorzuziehen.

\section{Literatur}

1. Mendel, B., Klin. Wschr. 8, 169 (1929). - 2. MeYerhof, O., K. LohmanN und Pr. Schuster, Biochem Z. 286,319 (1936). vergl. auch Embden, G., E. Schmitz und M. Wittenberg, Hoppe-Seyler's Z.physiol. Chem. 88, 210 (1913). 3.Lardy, H.A., V.D. Wiebelhaus und K.M. MANN, J. biol. Chemistry 187, 325 (1950). - 4. WArBuRG,
O. und G. Krippahl, Z. Naturforsch. 17b, 631, (1962); diese Z. 1, 33 (1963). - 5. WarBURG, O., Weiterentwicklung der zellphysiologischen Methoden, Georg Thieme Verlag, Stuttgart und Interscience, New York (1962).
Professor Dr. Otto Warburg Max-Planck-Institut für Zellphysiologie 1 Berlin 33, Garystr. 32 